\title{
Polymorphism of Interleukin 28 and HCV: Implementation of a method
}

\author{
Alessandra Flaminio, Olivia Turri, Antonella Rizzo, Antonella Morandi, Manuela Murano, \\ Claudia Eusebio, Roberta Marotta, Maria Luisa Biondi \\ UO Diagnostica Molecolare Infettivologica, Azienda Ospedaliera San Paolo, Milano
}

Key words: HCV, High resolution melting, IL28B

\section{Polimorfismo di Interleuchina 28 e HCV: Messa a punto di un metodo}

\section{SUMMARY}

$\mathrm{HCV}$ infection represents a global health problem. Only $20-30 \%$ of patients affected by HCV infection recovers spontaneously, while the remaining patients develop a chronic infection with risk of evolution to cirrhosis and hepatocarcinoma.

Nowadays, approximately $50 \%$ of individuals with hepatitis C infection is not responding to therapy with pegylated alphainterferon and ribavirin.

Polymorphisms (SNPs) of the gene coding for the interleukin 28 (IL28) have recently been described and they are in strong relationship with the outcome of HCV infected patients. In particular, the polymorphism rs $12979860(\mathrm{C} / \mathrm{T})$, located $3 \mathrm{~kb}$ upstream of the gene, is associated to a rapid and early response to therapy (genotype $\mathrm{C} / \mathrm{C}$ ).

Consequently we have developed a method in high resolution melting (HRM), which allows a simple and rapid screening of polymorphism rs 12979860.

The validation of the method was carried out by analyzing the IL28 genotype of 50 patients already determined by sequencing.

Sensitivity and specificity of the method were found to be equal to $100 \%$. Accuracy, precision within and between series were equal to $100 \%$. Compared to other methods described for the analysis of polymorphisms the HRM has the advantage of being faster and safe, relatively cheap and very simple in the optimization phase, therefore, applicable to a large throughput.

Up till now we have analyzed 329 patients of which 46 co-infected HCV-HIV.

The distribution of the polymorphism obtained is the following: $37 \% \mathrm{C} / \mathrm{C}, 5 \mathrm{I} \% \mathrm{C} / \mathrm{T}, 12 \% \mathrm{~T} / \mathrm{T}$. The distribution in the co-infected patients does not differ from that of the total HCV positive patients. We suggest a careful follow up of the therapeutic response of these patients to confirm the clinical usefulness of the test and to determine its true predictive value.

\section{INTRODUZIONE}

L'infezione da virus dell'epatite C (HCV) colpisce 170 milioni di persone in tutto il mondo (14). Di queste solo il 20-30\% guarisce spontaneamente; il rimanente sviluppa un'infezione cronica con rischio di evoluzione a cirrosi ed epatocarcinoma $(7,9)$. Il $50 \%$ dei pazienti affetti da epatite $\mathrm{C}$ cronica risulta non rispondere alla terapia con $\alpha$ interferone pegilato e ribavirina $(1,2,4,8,15)$.

Recentemente sono stati descritti due polimorfismi (SNPs) in associazione al gene codificante per l'interleuchina 28 (IL-28). In particolare il polimorfismo rs12979860 (C/T), situato $3 \mathrm{~kb}$ a monte del gene, risulta associato a una rapida e precoce risposta alla terapia (genotipo $\mathrm{C} / \mathrm{C})(3$, 10-13).

Scopo del nostro studio è quello di mettere a punto un metodo rapido e di semplice esecuzione per effettuare lo screening del polimorfismo rs12979860.

\section{MATERIALI E METODI}

Sono stati analizzati i campioni di sangue provenienti da 329 pazienti HCV positivi di cui 46 coinfetti con HIV.

Il DNA è stato estratto da leucociti di sangue periferico utilizzando il kit commerciale di estrazione QIAsymphony DNA mini kit.

I campioni sono stati analizzati attraverso High Resolution Melting (HRM) che consente un semplice e rapido screening del polimorfismo rs12979860 $(5,6)$.

L'analisi è stata effettuata su Rotor Gene Q (Qiagen) utilizzando una Master Mix HRM 1X (Qiagen), contenente: deossinucleotidi trifosfato (dNTPs), HRM PCR Buffer, Q solution e Eva

\section{Corresponding author: Maria Luisa Biondi}

U.O. Diagnostica Molecolare Infettivologica, Azienda ospedaliera San Paolo,

Via A. Di Rudinì 8 20I42 Milano, Italy - Tel. 028I8443|4 - Fax 028I844027

E-mail: marialuisa.biondi@ao-sanpaolo.it 
Green dye.

Per la PCR sono stati utilizzati 4 ng di DNA genomico in un volume finale di $10 \mu 1$. Per l'HRM sono stati aggiunti $5 \mu 1$ di Master Mix HRM $1 X$ e i primers 5'-CTGCACAGTCTGGGATTCCT3' e 5'AGGCTCAGGGGTCAATCACAG-3' (Invitrogen) (concentrazione finale $0.7 \mu \mathrm{M}$ ). Il profilo utilizzato per la PCR prevede uno step di denaturazione iniziale a $95^{\circ} \mathrm{C}$ per 5 secondi, seguito da 40 cicli così costituiti: denaturazione a $95^{\circ} \mathrm{C}$ per 10 secondi, annealing a $55^{\circ} \mathrm{C}$ per 30 secondi e allungamento a $72^{\circ} \mathrm{C}$ per 10 secondi, con l'acquisizione in fluorescenza a singolo canale.

Alla PCR fa seguito un'analisi di melting da $85^{\circ} \mathrm{C}$ a $95^{\circ} \mathrm{C}$ (Figura I).

La validazione del metodo è stata effettuata confrontando il genotipo di IL-28 di 50 pazienti precedentemente determinato tramite sequenziamento diretto (ABI Prism 310 - Applied Biosystems).

\section{RISULTATI}

Sensibilità e specificità del metodo sono risul- tate pari al $100 \%$ dal momento che non abbiamo riscontrato discordanze tra HRM e sequenziamento. La ripetibilità intra e interserie ottenuta è stata del $100 \%$.

Nei 329 pazienti analizzati la distribuzione del polimorfismo ottenuta è stata la seguente: $37 \%$ $\mathrm{C} / \mathrm{C}, 51 \% \mathrm{C} / \mathrm{T}, 12 \% \mathrm{~T} / \mathrm{T}$.

La distribuzione dei coinfetti non si discosta da quella della popolazione $\mathrm{HCV}$ positiva totale.

\section{DISCUSSIONE}

L'infezione da HCV rappresenta un problema sanitario globale.

Ci proponiamo un attento follow up della risposta terapeutica di questi pazienti per confermare l'effettiva utilità clinica del test e determinare il suo reale valore predittivo.

Rispetto ad altri metodi descritti per l'analisi di polimorfismi l'HRM ha il vantaggio di essere più rapido e sicuro, relativamente poco costoso e molto semplice nella fase di ottimizzazione, quindi applicabile ad un ampio throughput.

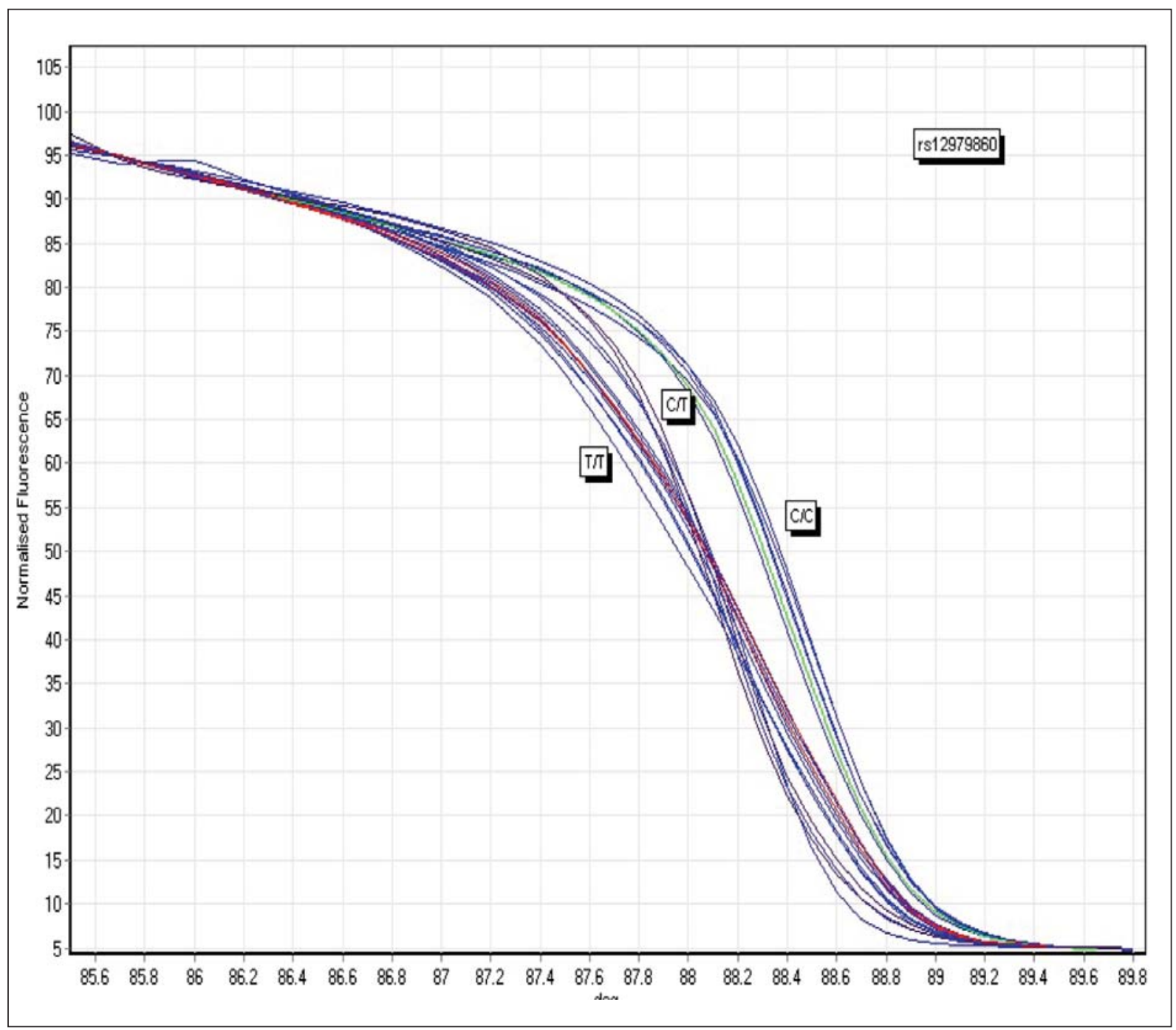

Figura I. HRM for IL28B polymorphisms normalized temperature-shifted melting curves. 


\section{BIBLIOGRAFIA}

1. Chung RT, Andersen J, Volberding $P$, et al. Peginterferon alfa-2a plus ribavidin versus interferon alfa-2a plus ribavirin for chronic hepatitis C in HIVcoinfected persons. N Engl J Med 2004; 351: 451-9.

2. Dore GJ, Hellard M, Matthews GV, et al. Effective treatment of injecting drug users with recently acquired hepatitis C virus infection. Gastroenterology 2010; 138: 123-35.

3. Ge D, Fellay J, Thompson AJ, et al. Genetic variation in IL28B predicts hepatitis C treatment induced viral clearance. Nature 2009; 461: 399-401.

4. Grebely J, Matthews GV, Petroumenos K, Dore GJ. Spontaneous clearance and beneficial impact of treatment on clearance during recent hepatitis $\mathrm{C}$ virus infection. J Viral Hepat 2010; 17: 896.

5. Herrmann MG, Durtschi JD, Bromley LK, Wittwer CT, Voelkerding KV. Amplicon DNA melting analysis for mutation scanning and genotyping: cross-platform comparison of instruments and dyes. Clin Chem 2006; 52: 494-503.

6. Herrmann MG, Durtschi JD, Wittwer CT, Voelkerding $\mathrm{KV}$. Expanded instrument comparison of amplicon DNA melting analysis for mutation scanning and genotyping. Clin Chem 2007; 53: 1544-8.

7. Hoofnagle JH. Course and outcome of hepatitis C. Hepatology 2002; 36: S21-9.
8. Kamal SM, Fouly AE, Kamel RR, et al. Peginterferon alfa-2b therapy in acute hepatitis C: impact of therapy on sustained virologic response. Gastroenterology 2006; 130: 632-8.

9. Micallef JM, Kaldor JM, Dore GJ. Spontaneous viral clearance following acute hepatitis $\mathrm{C}$ infection: a systematic review of longitudinal studies. $J$ Viral Hepat 2006; 13: 34-41.

10. Rauch A, Kutalik Z, Descombes P, et al. Genetic variation in IL28B is associated with chronic hepatitis C and treatment failure: a genome wide association study. Gastroenterology 2010; 138: 1240-3.

11. Suppiah V, Moldovan M, Ahlenstiel G, et al. IL28B is associated with response to chronic hepatitis $\mathrm{C}$ interferon-alpha and ribavirin therapy. Nat Genet 2009; 41: 1100-4.

12. Tanaka Y, Nishida N, Sugiyama M, et al. Genomewide association of IL28B with response to pegylated interferon-alpha and ribavirin therapy for chronic hepatitis C. Nat Gen 2009; 41: 1105-9.

13. Thomas DL, Thio CL, Martin MP, et al. Genetic variation in IL28B and spontaneous clearance of hepatitis C virus. Nature 2009; 461: 798-801.

14. WHO. Hepatitis C. http://www.who.int/medioacentre/factsheets/fs164/en/ (October 2000).

15. Zeuzem S, Feinman SV, Rasenack J, et al. Peginterferon alfa-2a in patients with chronic hepatitis C. N Engl J Med 2000; 343: 1666-72. 